\title{
The histologic presentation of hepatitis E reflects patients' immune status and pre-existing liver condition
}

\author{
Daniela Lenggenhager ${ }^{1}$ - Samuel Pawel ${ }^{2} \cdot$ Hanna Honcharova-Biletska ${ }^{1}$ Katja Evert ${ }^{3}$ Jürgen J. Wenzel $\oplus^{4}$ •

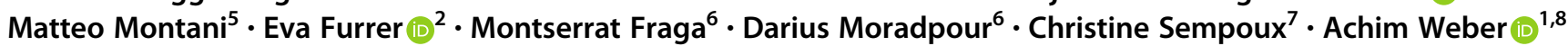

Received: 9 March 2020 / Revised: 27 May 2020 / Accepted: 27 May 2020 / Published online: 22 June 2020

(c) The Author(s) 2020

\begin{abstract}
Infection with the hepatitis $\mathrm{E}$ virus (HEV) is one of the main causes of acute hepatitis worldwide. Given that, the histopathology of hepatitis $\mathrm{E}$ is relatively poorly characterized, and it is unclear what exactly determines its remarkable variability. The aim of our study was a systematic analysis of hepatitis E histology, especially with regard to the clinical setting. Fifty-two liver samples (48 biopsies, 1 liver explant, 3 autopsy livers) from 41 patients with molecularly proven hepatitis E (28 HEV genotype (gt) 3, three gt 1, one gt 4 and 9 undetermined gt) were systematically evaluated for 33 histopathologic features. Following one approach, the biopsies were assigned to one of five generic histologic patterns. In another approach, they were subjected to hierarchical clustering. We found that $23 / 41$ (56\%) patients were immunocompromised, whereas 18 (44\%) had no known immunosuppression. Five patients $(12 \%)$ had pre-existing liver disease (LD). The histopathologic spectrum ranged from almost normal to acute, chronic, and steato-hepatitis to subtotal necrosis, and was thus distributed across all five generic patterns. Hierarchical clustering, however, identified three histopathologic clusters (C1-C3), which segregated along the immune status and pre-existing LD: C1 comprised mostly patients with pre-existing LD; histology mainly reflected the respective LD without pointing to the additional hepatitis E. C2 comprised mostly immunocompetent patients; histology mainly displayed florid hepatitis. C3 comprised mostly immunocompromised patients; histology mainly displayed smoldering hepatitis. Accordingly, C1-C3 differed markedly with respect to their clinical and histopathologic differential diagnoses. Hierarchical clustering suggests three groups with distinct histopathologies, indicating biologically different manifestations of hepatitis E. The association of histopathologic changes with the patient's immune status and pre-existing LD plausibly explains the diversity of hepatitis E histopathology, and suggests that these factors are the crucial underlying determinants. We expect our results to improve patient management by guiding the clinico-pathologic diagnosis of hepatitis $\mathrm{E}$.
\end{abstract}

Supplementary information The online version of this article (https:// doi.org/10.1038/s41379-020-0593-1) contains supplementary material, which is available to authorized users.

Achim Weber

achim.weber@usz.ch

1 Department of Pathology and Molecular Pathology, University of Zurich and University Hospital Zurich, Zurich, Switzerland

2 Epidemiology, Biostatistics and Prevention Institute, University of Zurich, Zurich, Switzerland

3 Institute of Pathology, University of Regensburg, Regensburg, Germany

4 Institute of Clinical Microbiology and Hygiene, Regensburg University Medical Center, University of Regensburg,

\section{Introduction}

Hepatitis E is caused by a water- or food-borne, enterically transmitted infection with the hepatitis E virus (HEV), a non-enveloped, positive-strand RNA virus $[1,2]$. HEV

Regensburg, Germany

5 Institute of Pathology, University of Bern, Bern, Switzerland

6 Division of Gastroenterology and Hepatology, Centre Hospitalier Universitaire Vaudois, University of Lausanne,

Lausanne, Switzerland

7 Institut Universitaire de Pathologie, Centre Hospitalier Universitaire Vaudois, University of Lausanne, Lausanne, Switzerland

8 Institute of Molecular Cancer Research (IMCR), University of Zurich, Zurich, Switzerland 
infection is one of the most common causes of acute hepatitis worldwide and thus represents a major global health problem [3, 4]. The disease shows clear differences between resource-rich and resource-limited countries in terms of viral genotypes (gt), mode of transmission and course of disease. Resource-limited countries are dominated by gt 1 and gt 2 , which occur endemically as well as epidemically, are transmitted from humans to humans predominantly by contaminated drinking water and occasionally cause large epidemic outbreaks. By contrast, resource-rich countries are dominated by zoonotic transmission of HEV gt 3, which occurs worldwide, as well as gt 4, which is mainly found in China and Southeast Asia. Transmission of gt 3 and gt 4 often occurs through contaminated meat products [5].

The clinical course of hepatitis $\mathrm{E}$ is highly variable. HEV gt 3 infection is usually asymptomatic or subclinical but can also present as acute, self-limiting hepatitis, or even as acute-on-chronic liver failure in patients with pre-existing liver disease $[3,6]$. Poor outcomes have also been described in pregnant women infected with HEV gt 1 $[3,6]$. Originally regarded as an exclusively acute infection, the experience of the last decade has shown that hepatitis E of gt 3 can also develop a chronic course under immunosuppression [7].

Shortly after a peculiar form of epidemic hepatitis, later on designated as hepatitis $\mathrm{E}$, had been identified as a distinct disease in India in the mid-fifties [8], the histologic changes observed in $>70$ cases of this outbreak were described [9]. For a long time, these reports, limited to the epidemic form of hepatitis $\mathrm{E}$ and enriched for severe courses, were the only ones available. Several decades later, the first histologic descriptions of autochthonous hepatitis $\mathrm{E}$ were published in smaller series [10-13], including HEV infection taking a chronic course [7, 14-16]. Although a comprehensive, comparative analysis of histology, especially with regard to characteristics of the host, is still lacking, from the body of literature on hepatitis $\mathrm{E}$ histology it is obvious that findings are highly variable and overlapping with other causes of hepatitis, thus, making a timely and accurate histopathologic diagnosis of HEV infection challenging [10, 14, 17-19]. Since the histologic changes reflect the interaction between the virus on the one hand and the patient on the other, we here have aimed at a systematic analysis of hepatitis $\mathrm{E}$ histology and its association with clinical parameters.

\section{Materials and methods}

\section{Patients and liver tissue samples}

Included into this study were 52 liver specimens (48 biopsies, 1 liver explant, and 3 autopsy livers) which have been analyzed at the Department of Pathology and Molecular Pathology, University Hospital Zurich (USZ) between 2012 and 2018. The cohort comprised cases primarily submitted to the USZ and cases referred to us from other centers, including the Cantonal Hospitals St. Gallen and Lucerne, the University Hospitals Lausanne, Geneva, Basel and Bern, all Switzerland, the University Hospital Regensburg, Germany, and the Massachusetts General Hospital, Boston, MA, USA. For this study, all liver specimens submitted to our center were included from patients who had molecularly confirmed HEV infection $(n=41)$, i.e., having a positive HEV RNA PCR in blood and/or tissue. Two or more specimens taken at different time points were analyzed from eight of the 41 patients. Information on demographic, clinical, and virological parameters, including age, sex, immune status, pre-existing LD, and viral genotype, was retrieved retrospectively from the patient charts. This study was approved by the internal review board of the USZ and the Cantonal Ethics Committee of Zurich, Switzerland (KEK-ZH-Nr. 2013-0504).

\section{Histopathologic evaluation of liver biopsies}

All liver specimens were processed according to standard histologic methods. Stains included hematoxylin \& eosin (H\&E) and a connective tissue stain (such as Sirius red or Masson trichrome stain) for all cases, as well as an additional periodic acid-Schiff stain after diastase digestion (PAS-D). Histologic slides were re-evaluated independently by three experienced liver pathologists (DL, CS, AW). For all biopsies, the following 33 histopathologic features were systematically recorded and graded: portal inflammation (0-3), portal lymphocytes (0-2), portal plasma cells $(0-2)$, portal histiocytes $(0-2)$, portal ceroid-laden histiocytes (0-2), portal neutrophils (0-2), portal eosinophils (0-2), portal edema (no/yes), bile duct damage (0-2), ductular reaction (no/yes), interface activity (0-3), lobular inflammation (0-3), hepatocyte necrosis (0-3), lobular ceroidladen histiocytes (0-2), Kupffer cell activation (0-2), lobular disarray (0-2), rosettes (0-3), anisonucleosis (no/yes), hepatocyte damage (0-2), Mallory-Denk hyaline (no/yes), sinusoidal dilatation (no/yes), cholate stasis (no/yes), bilirubinostasis (0-2), steatosis microvesicular (0-2), steatosis macrovesicular (no/yes), Brunt score for steatosis (0-3) [20], fibrosis (0-3), sinusoidal fibrosis (no/yes), activity according to the classifications METAVIR (A0-A3) [21], and Batts-Ludwig (A0-A4) [22] as well as fibrosis according to the classifications METAVIR (F0-F4) [21], Batts-Ludwig (F0-F4) [22], and Ishak (0-6) [23] (Supplementary Table 1).

According to this evaluation, each specimen was assigned to one of the following five generic histologic patterns by all three pathologists: (1) Minimal active hepatitis, (2) 
Table 1 Cohort characteristics: patients and liver specimens.

\begin{tabular}{|c|c|}
\hline Patients, $n$ & 41 \\
\hline Median age at first biopsy (range) & $56(10-76)$ \\
\hline Sex male/female, $n(\%)$ & $27 / 14(66 \% / 34 \%)$ \\
\hline HEV genotype (gt), $n(\%)$ & $\begin{array}{l}\text { - gt 1: } 3(7.3 \%) \\
\text { - gt 3: } 28(68.3 \%) \\
\text { - gt 4: } 1(2.4 \%) \\
\text { - NA: } 9(22.0 \%)\end{array}$ \\
\hline \multicolumn{2}{|l|}{ Immune status } \\
\hline - Immunocompetent, $n(\%)$ & $18 / 41(44 \%)$ \\
\hline No known pre-existing LD, $n(\%)$ & $-13 / 41(32 \%)$ \\
\hline O Pre-existing LD, $n(\%)$ & $-5 / 41(12 \%)$ \\
\hline - cirrhosis on ASH & $\bigcirc$ \\
\hline - cirrhosis on NASH & $\bigcirc 1$ \\
\hline - cirrhosis on ASH/NASH & $\bigcirc 1$ \\
\hline - Immunocompromised, $n(\%)$ & $23 / 41(56 \%)$ \\
\hline TPL (solid organs or HSC), $n(\%)$ & $-14 / 41(34 \%)$ \\
\hline - Liver, $n$ & $\bigcirc 7$ \\
\hline - Kidney, $n$ & $\bigcirc 2$ \\
\hline - Liver and kidney, $n$ & $\bigcirc 1$ \\
\hline - HSC, $n$ & $\bigcirc 4$ \\
\hline Otherwise, $n(\%)$ & $-9 / 41(22 \%)$ \\
\hline - RA (methotrexate \pm infliximab) & $\bigcirc 2$ \\
\hline - MS (fingolimod) & $\bigcirc 2$ \\
\hline $\begin{array}{l}\text { - SLE and cryoglobulinemia (prednisolone } \\
\text { and leflunomide) }\end{array}$ & $\bigcirc 1$ \\
\hline $\begin{array}{l}\text { - AIH (prednisone or prednisolone and } \\
\text { tacrolimus) }\end{array}$ & $\bigcirc 2$ \\
\hline - CLL (rituximab, leflunomide) & $\bigcirc 1$ \\
\hline - Histiocytosis X (chemotherapy NOS) & $\bigcirc 1$ \\
\hline Liver specimens, $n$ & 52 \\
\hline - Biopsy, $n(\%)$ & $-48 / 52(92 \%)$ \\
\hline - Explant liver, $n(\%)$ & $-1 / 52(2 \%)$ \\
\hline - Autopsy liver, $n(\%)$ & $-3 / 52(6 \%)$ \\
\hline
\end{tabular}

AIH autoimmune hepatitis, $A S H$ alcoholic steatohepatitis, $C L L$ chronic lymphocytic leukemia, gt genotype, $H E V$ hepatitis E virus, $H S C$ hematopoietic stem cells, $L D$ liver disease, $M S$ multiple sclerosis, $N A$ not available, NASH nonalcoholic steatohepatitis, NOS not otherwise specified, $R A$ rheumatoid arthritis, SLE systemic lupus erythematosus, $T P L$ transplantation.

Predominantly lobular hepatitis, (3) Predominantly portal hepatitis, (4) Steatohepatitis, and (5) Extensive necrosis (pattern definition see Supplementary Table 2). In case of discrepancies, consensus was found in a panel discussion.

\section{Statistics}

To quantify the inter-rater agreement of the assessments of the histopathologic features, the weighted kappa statistic was computed using quadratic weights. For the histologic pattern variables, the usual kappa statistic was used [24]. Divisive hierarchical clustering of the 33 histopathologic features was conducted according to the algorithm by Kaufman and Rousseeuw [25]. Since the histopathologic features were on ordinal scales with different ranges and a few values were missing, an extension of Gower's generalized dissimilarity coefficient to compute dissimilarities for the clustering procedures was used (with the algorithm "daisy" available in the R-package "cluster") [25]. Gower's method also allows for different weighting of features. We assigned a weight of 1 to all features, except the following: Steatosis macrovesicular and Brunt-Score for steatosis received a weight of $1 / 2$ each, activity METAVIR and activity Batts-Ludwig received a weight of $1 / 2$ each, and fibrosis METAVIR, fibrosis Batts-Ludwig, and fibrosis Ishak received a weight of $1 / 3$ each. This was done to avoid overweighting these features in the dissimilarity calculation as they are measures of the same histologic parameters [25]. Finally, the results of the cluster analysis were then displayed in a heatmap, in which the histopathologic features have been properly scaled such that criteria with different number of categories can be compared. All statistical analyses were performed in the $\mathrm{R}$ programming language [26] using base packages, as well as the psy [27], cluster [28], gplots [29], and ved [30] packages.

\section{Results}

\section{The cohort comprises mostly HEV genotype 3 infections in immunocompetent as well as immunocompromised individuals}

Basic demographic and clinical characteristics as well as the results of genotyping were retrospectively retrieved from the charts of all 41 patients. These are summarized in Table 1 and listed in more detail in Table 2. From eight patients, there were two or more liver samples available over time, yielding a total of 52 liver specimens for analysis. The age at the first liver biopsy ranged from 10 to 76 years, with a median age of 56 years. Twenty-seven patients (66\%) were male and 14 (34\%) were female. HEV infection was proven molecularly by positive HEV RNA PCR in blood and/or tissue in all 41 patients. In 32 of these 41 patients, the HEV gt was determined, with the following distribution: gt 3 in 28 patients $(68.3 \%)$, gt 1 in three patients $(7.3 \%)$, and gt 4 in one patient $(2.4 \%)$. For nine patients $(22.0 \%)$, no information on the HEV genotype was available.

Four different patient groups were represented based on clinical considerations [3, 4]. These comprised immunocompetent individuals without or with known pre-existing 


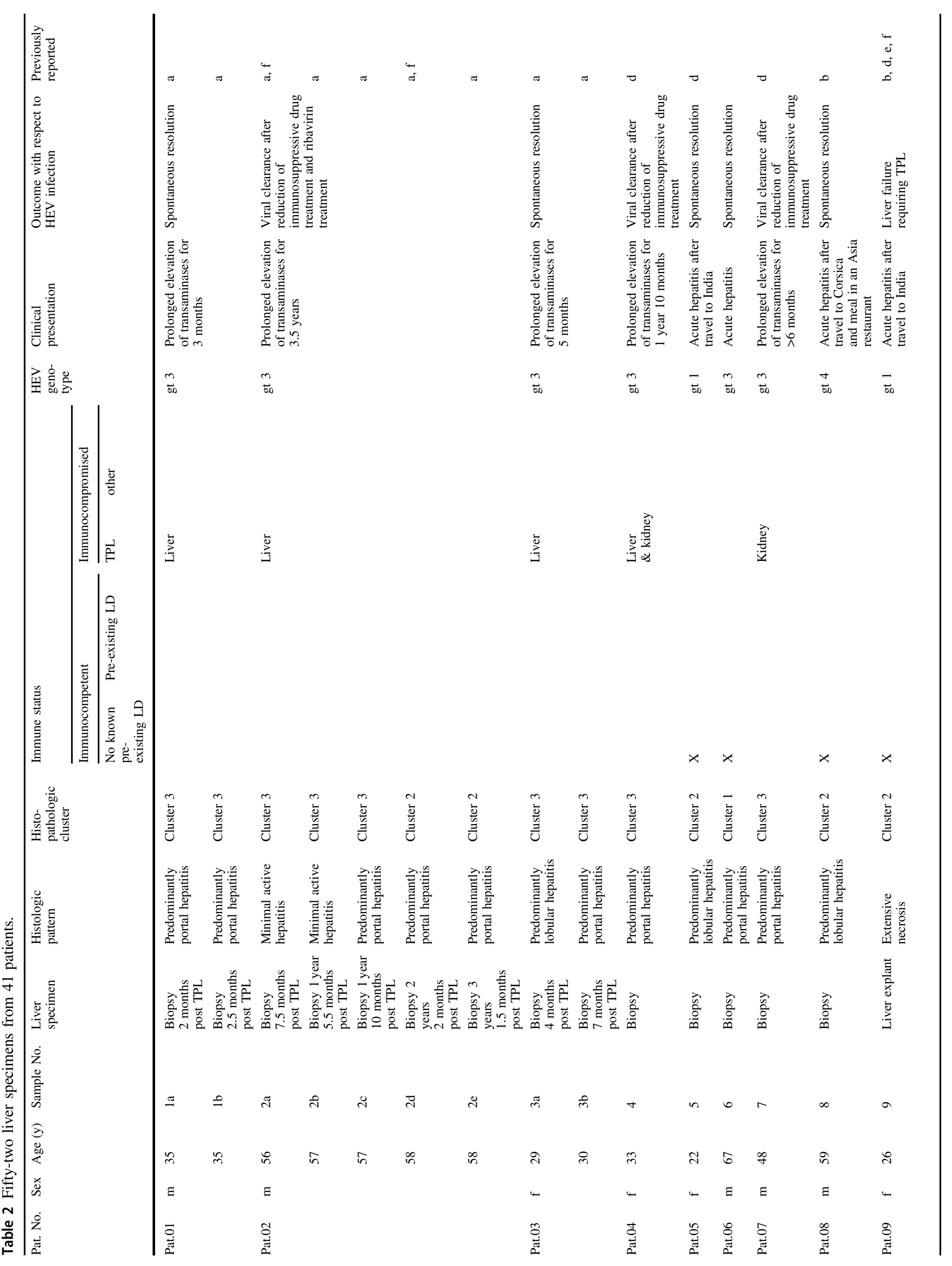




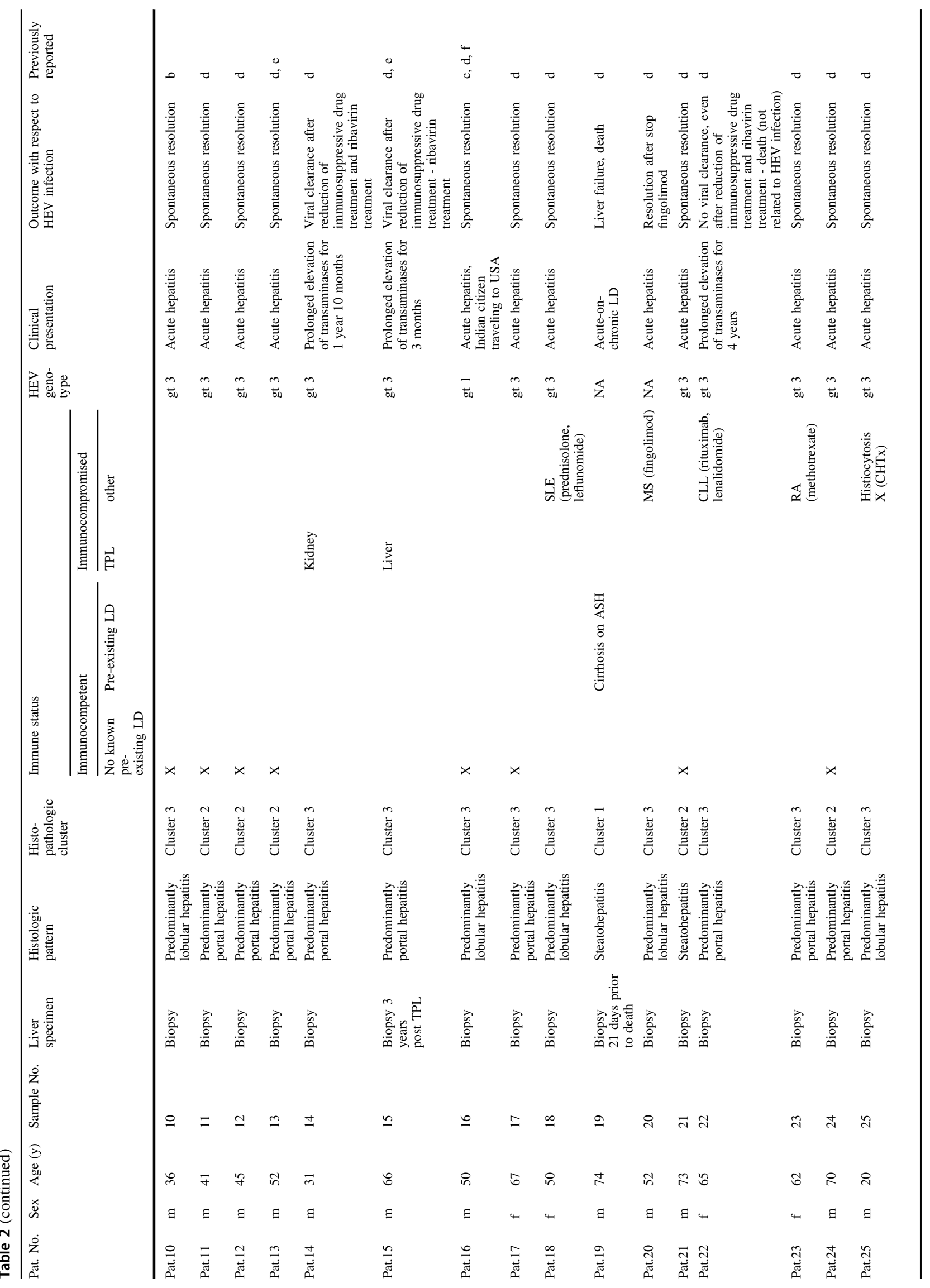




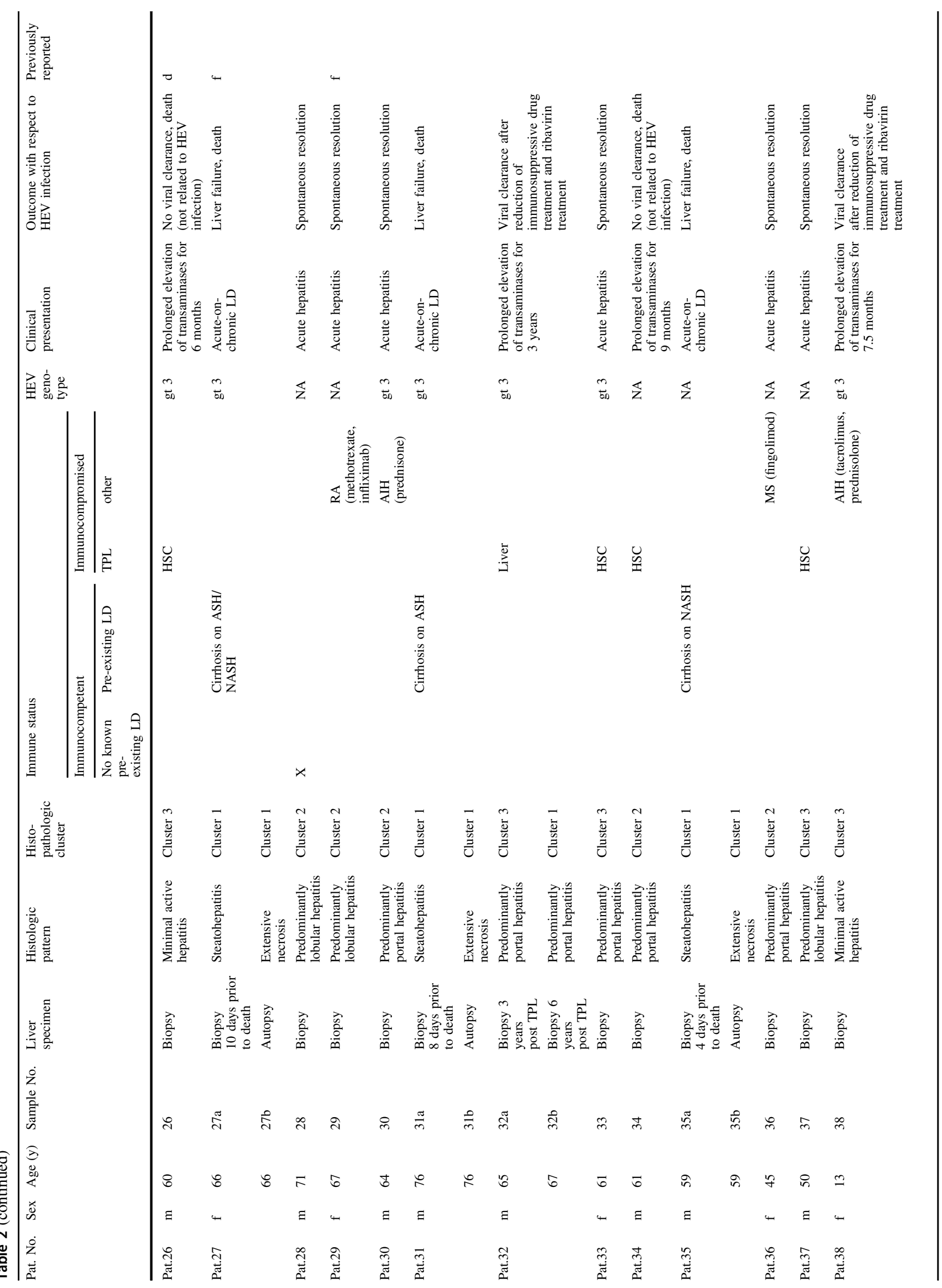




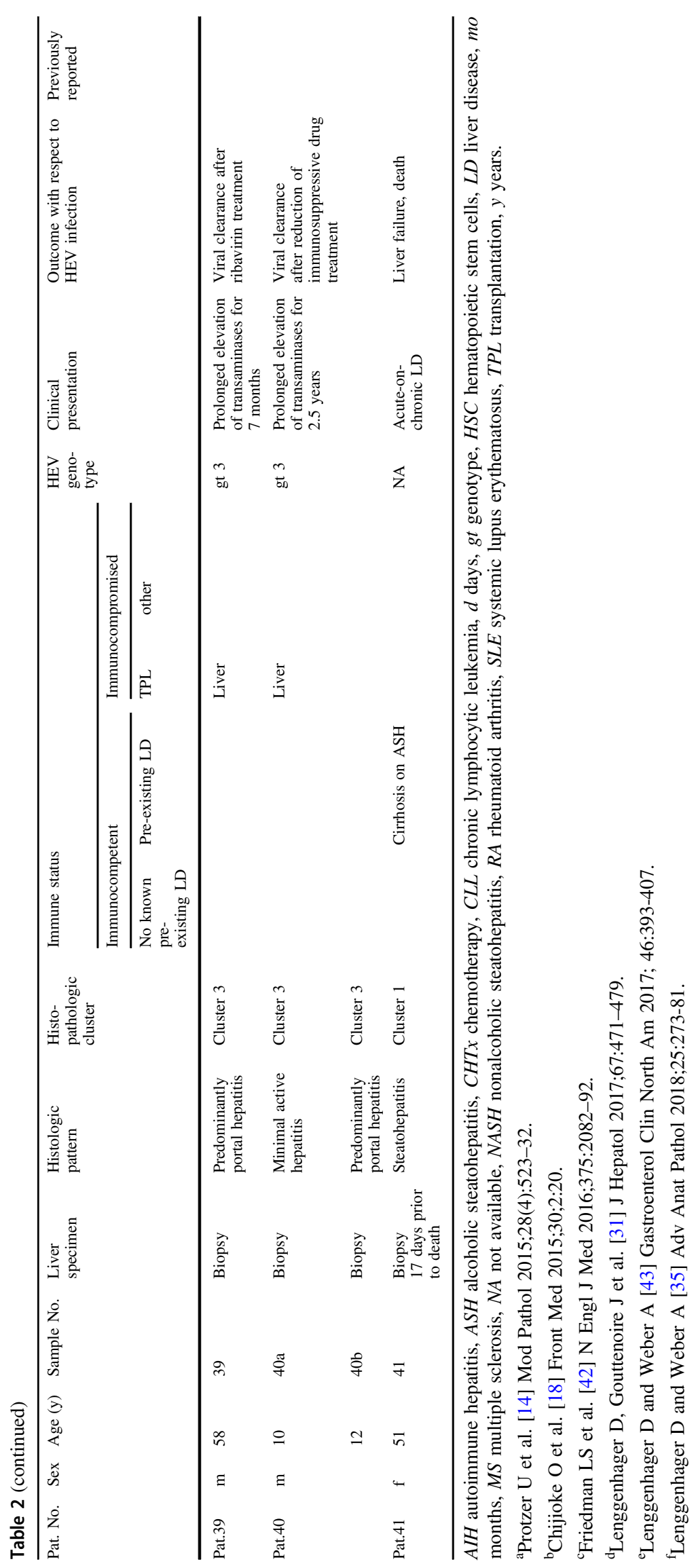


LD and immunocompromised patients after transplantation (solid organ or hematopoietic stem cell (HSC)), or otherwise immunocompromised. With respect to these categories, 18 of the 41 patients were immunocompetent (43.9\%), with a total of 21 liver specimens $(21 / 52 ; 40.4 \%)$. Of these 18 patients, 13 did not have any known preexisting LD $(13 / 41 ; 31.7 \%)$, with a total of 13 liver specimens $(13 / 52 ; 25.0 \%)$, and five patients had a known pre-existing $\operatorname{LD}(5 / 41 ; 12.2 \%)$, with a total of eight liver specimens $(8 / 52 ; 15.4 \%)$. All five patients with known preexisting LD had cirrhotic livers. Etiologies of the cirrhosis included ASH (3/5), NASH (1/5), or ASH/NASH (1/5). Twenty-tree patients were immunocompromised (23/41; $56.1 \%)$, with a total of 31 liver specimens $(31 / 52 ; 59.6 \%)$. The group of immunocompromised patients comprised 14 patients after solid organ or HSC transplantation (14/41; $34.1 \%)$, with totally 22 liver specimens $(22 / 52 ; 42.3 \%)$, and nine otherwise immunocompromised patients $(9 / 41$; $22.0 \%)$, with totally nine liver specimens $(9 / 52 ; 17.3 \%)$. Of the 14 transplanted patients, seven received a liver (7/14), two a kidney (2/14), one a liver and a kidney (1/14), and four HSC (4/14). Patients immunocompromised for other reasons included treatment for rheumatologic/autoimmune diseases (i.e., rheumatoid arthritis, multiple sclerosis, systemic lupus erythematosus, autoimmune hepatitis) or neoplasia (i.e., chronic lymphocytic leukemia, histiocytosis $\mathrm{X}$ ).

\section{A broad and highly variable spectrum of histopathologic findings can be observed in livers of patients infected with HEV}

First, all 52 liver specimens were re-evaluated independently by three expert liver pathologists, each recording systematically 33 histopathologic features (Supplementary Table 1). Inter-rater agreement in this independent evaluation was between good to excellent, with kappa values between 0.4 and 0.9 (two-thirds $\geq 0.75$ ) for most histopathologic features (Supplementary Fig. 1). The microscopic review revealed a wide range of histopathologic findings, which varied significantly from case to case. This spectrum of hepatitis E histology is illustrated in Supplementary Fig. 2a-e. To corroborate the HEV infection on a tissue level, immunohistochemistry was performed in cases with sufficient block material, which was possible in a total of 40 tissue samples. Various staining patterns were observed as previously described [31]. Remarkably, cases with only minimal lobular inflammatory activity usually showed the strongest and most widespread positivity for HEV ORF2 protein (Supplementary Fig. 2f).

Collectively, these findings demonstrate that on the level of their histopathologic manifestation, the hepatitis E cases of the cohort were highly variable.

\section{Hepatitis E presents in five different generic histologic patterns}

Next, we aimed to assign a generic histologic pattern to each liver specimen. Such patterns are considered to reflect the limited repertoire of the liver to respond to injuries and are widely used in routine histopathologic practice to get direction for the underlying etiology and correct diagnosis [32]. The cases of our cohort revealed the following distribution among the five histologic patterns (Fig. 1): (1) Minimal active hepatitis: 5/52 (9.6\%; Fig. 1a), (2) Predominantly lobular hepatitis: $11 / 52$ (21.2\%; Figs. 1b), (3) Predominantly portal hepatitis: 26/52 (50.0\%; Figs. 1c), (4) Steatohepatitis: $6 / 52$ (11.5\%; Fig. 1d) and (5) Extensive liver necrosis: $4 / 52$ (7.7\%; Fig. 1e). Thus, similar as observed for the individual histopathologic features, also the histologic patterns displayed a broad distribution among the cases of our cohort (Table 2 and Supplementary Fig. 3a). Again, the inter-rater agreement for those patterns was excellent (kappa value $\geq 0.75$ ) (Supplementary Fig. 1). Of note, several of the histologic patterns were unequally associated with specific patient characteristics, some of them with statistical significance. For example, in the group of patients with pre-existing LD, the histologic patterns "steatohepatitis" and "extensive necrosis" were statistically significantly over-represented, whereas the pattern of "predominantly portal hepatitis" was statistically significantly under-represented (Supplementary Fig. 3b).

Collectively, these findings demonstrate that the histopathologic manifestation of hepatitis $\mathrm{E}$ covered a broad spectrum of well-established generic histologic patterns, some of which were preferentially associated with specific patient characteristics.

\section{Hierarchical clustering of 33 histopathologic features identifies three histopathologic clusters of hepatitis $\mathbf{E}$}

With the aim of uncovering any structure that may exist in this rich data set, regardless of any predetermined categories such as the patterns previously studied, we then decided to take a systematic approach by performing a cluster analysis. The principle of this analysis was to identify clusters of samples in which the samples within each cluster were histologically similar, but different from those in the other clusters. Specifically, we conducted hierarchical clustering of the samples with respect to the 33 histopathologic features recorded, as well as the other way around. Remarkably, this approach displayed an easy to identify structure in the data set, revealing three clusters of samples (C1-C3) with distinct histopathology. The liver samples were distributed as follows: Ten of the 
Fig. 1 Histopathologic findings observed in hepatitis $\mathbf{E}$ represented in five generic histologic patterns. a Mimimal active hepatitis with normal architecture, minimal portal, and lobular inflammation and only single scattered hepatocyte apoptosis. b Predominantly lobular hepatitis with prominent lobular disarray, rosettes formation, bilirubinostasis, hepatocyte damage, necrotic hepatocytes, bile duct damage, signs of beginning removal with lobular ceroid-laden histiocytes (PAS stain) and some parenchymal collapse (Sirius red stain). c Predominantly portal inflammation with preserved lobular architecture and some interface and lobular inflammatory activity with few hepatocyte apoptoses.

Developing fibrosis possible (Sirius red stain). d Cirrhotic parenchyma (Sirius red stain) with steatosis,

necroinflammation, hepatocyte ballooning, Mallory-Denk hyaline, bilirubinostasis and pronounced pericellular fibrosis (Sirius red stain). e Subtotal necrosis of liver parenchyma shown here in a cirrhotic liver (CAB stain). H\&E stain, if not otherwise specified. Scale bars overviews $400 \mu \mathrm{m}$, scale bars details $50 \mu \mathrm{m}$.
A
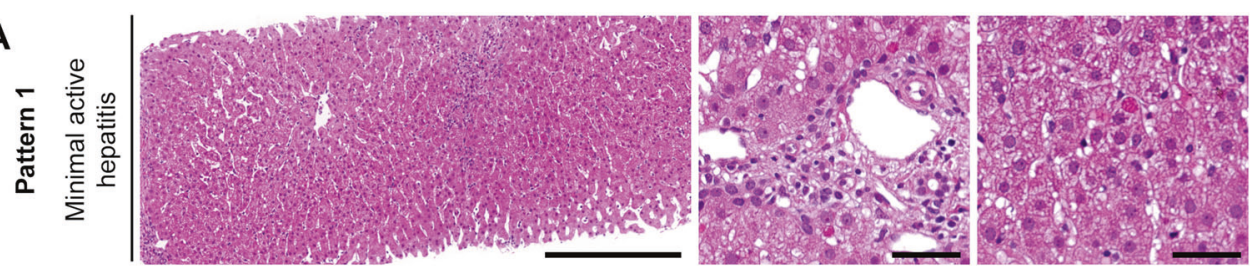

B
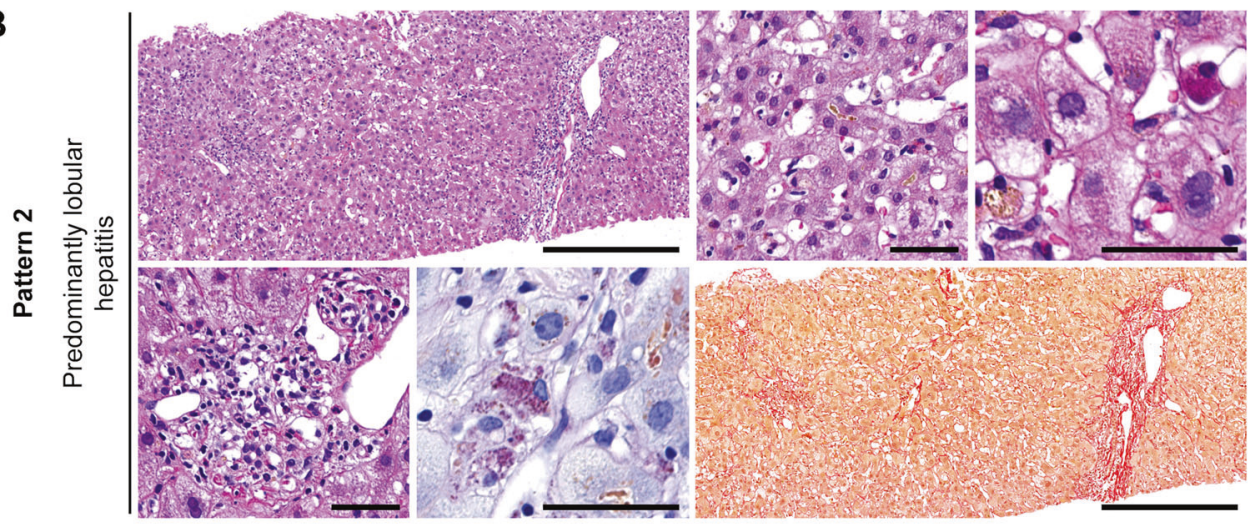

C

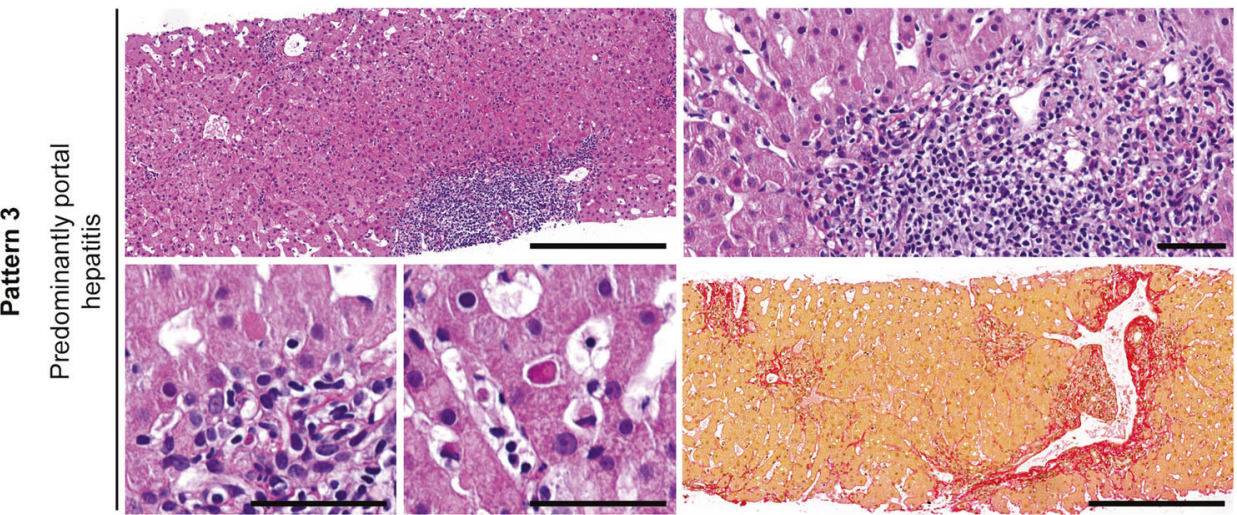

D
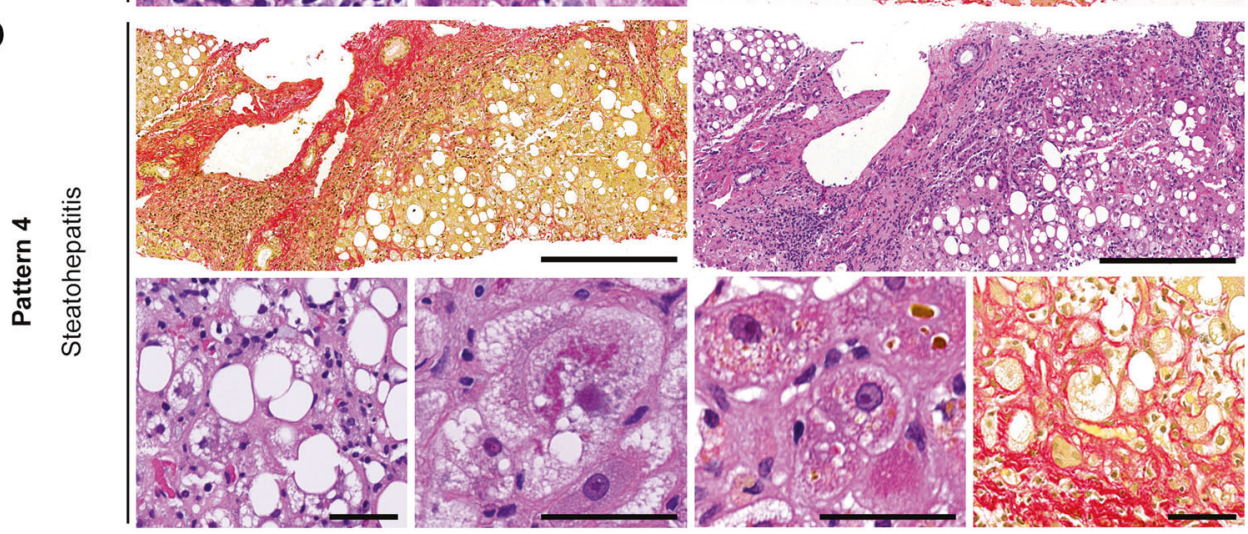

E
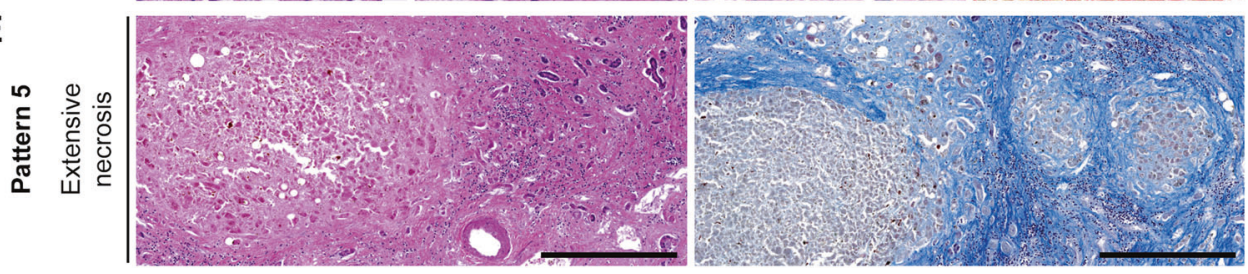

52 samples (19.2\%) in $\mathrm{C} 1,15$ samples (28.8\%) in $\mathrm{C} 2$ and 27 samples (52\%) in C3 (Fig. 2). The 33 histopathologic features were clearly represented differently in the three clusters. Of note, they were not randomly distributed, but clustered mainly according to the different pathologies they reflect (i.e., from top to bottom: features of acute 


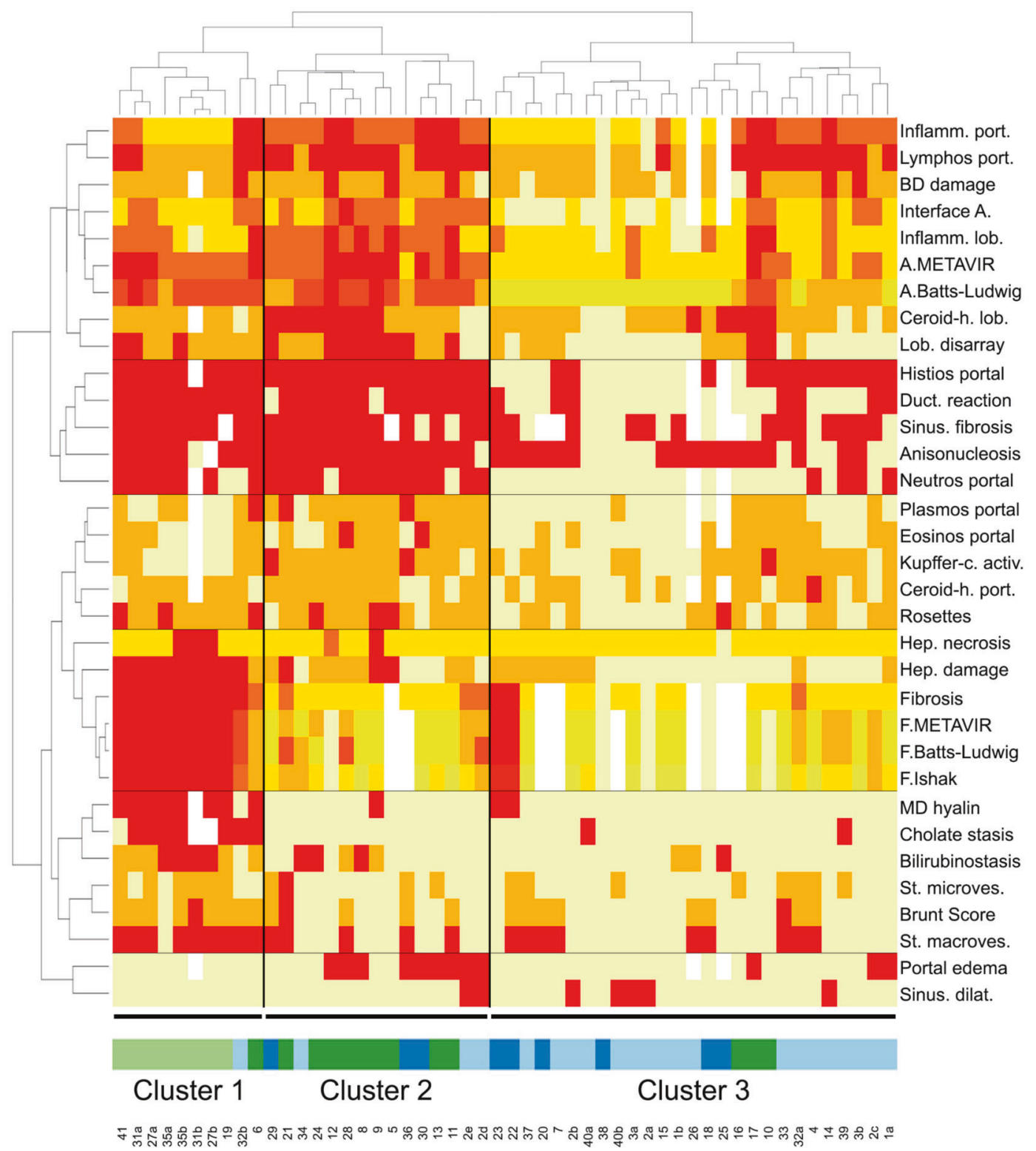

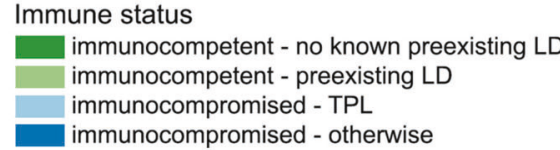

Fig. 2 Hierarchical clustering of histopathologic findings. Hierarchical clustering of the 52 liver specimens ( $x$ axis) with respect to the 33 histopathologic features ( $y$ axis) represented in a heatmap: Identification of three clusters $(\mathrm{C} 1-\mathrm{C} 3)$ that segregate along the patient's immune status and pre-existing LD. The 33 histopathologic features clustered mainly according to the different pathologies they reflect (i.e., from top to bottom: features of acute inflammatory activity, features of early subacute inflammatory activity, features of late

inflammatory activity, features of early subacute inflammatory activity, features of late subacute inflammatory activity, features of chronicity and features of cellular damage). Only two features (portal edema and sinusoidal

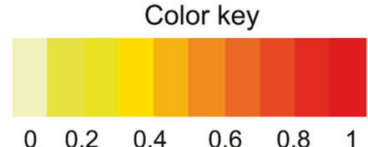

subacute inflammatory activity, features of chronicity and features of cellular damage). Only two features (portal edema and sinusoidal dilatation) did not cluster in any of the above mentioned groups. A activity, BD bile duct, Ceroid-h. Ceroid-histiocytes, Duct. ductular, F fibrosis, Hep. hepatocyte, Inflamm. inflammation, Kupffer-c. activ. Kupffer cell activation, LD liver disease, lob. lobular, MD MalloryDenk, port. portal, Sinus. sinusoidal, St. steatosis, TPL transplantation.

dilatation) did not cluster in any of the above mentioned groups (Fig. 2).

Each of the three clusters was characterized by a predominance of different histopathologic features (Fig. 3): 
Fig. 3 Histopathologic findings observed in hepatitis $\mathbf{E}$ represented in three histopathologic clusters. a Samples in C1 showed mainly features of acute-on-chronic LD, such as cirrhosis (Masson trichrome stain), necrosis, steatosis, steatohepatitis, hepatocyte damage, MalloryDenk hyaline and bilirubinostasis (from upper left to lower right). b Samples in C2 displayed mainly features of florid hepatitis, such as lobular inflammation and parenchymal disarray, foci of hepatocyte necrosis and many scattered apoptotic hepatocytes as well as signs of beginning removal with lobular ceroid-laden histiocytes (PAS-D stain), cholestasis and bile duct damage (from upper left to lower right). c Samples in C3 were mainly characterized by features of smoldering hepatitis, ranging from minimal histologic changes over predominantly portal based inflammation with mild interface and lobular activity with only rare apoptotic hepatocytes as well as signs of previous damage such as ceroidladen histiocytes (PAS-D stain) in portal tracts and in some cases with fibrosis (Sirius red stain) (from upper left to lower right). H\&E stain, if not otherwise specified. Scale bars overviews $400 \mu \mathrm{m}$, scale bars details 50 $\mu \mathrm{m}$. C cluster, LD liver disease.
A
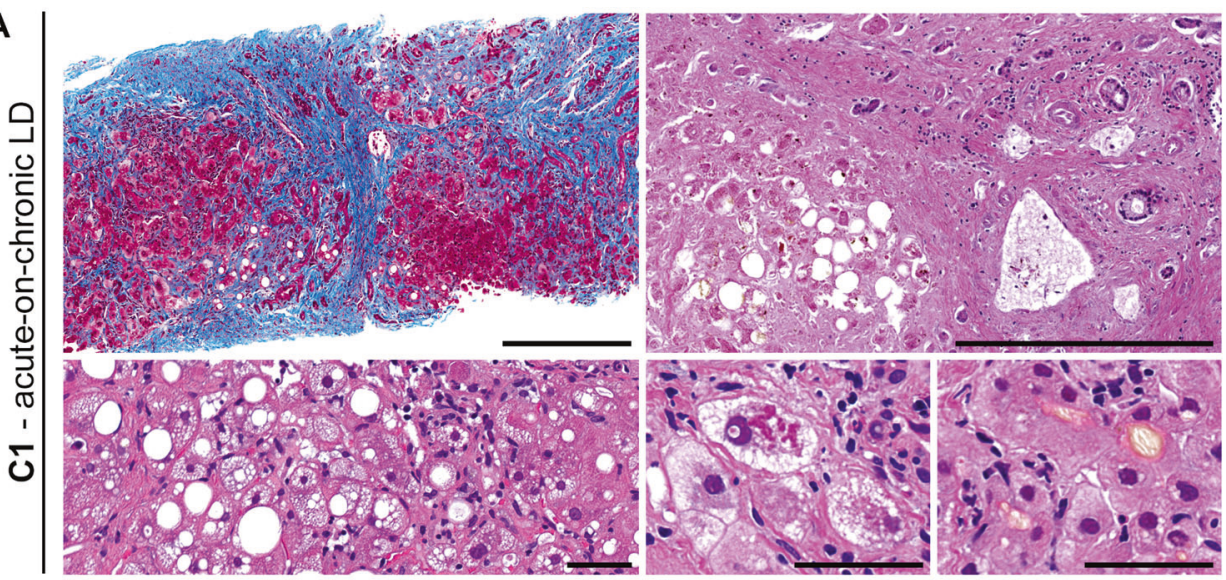

B
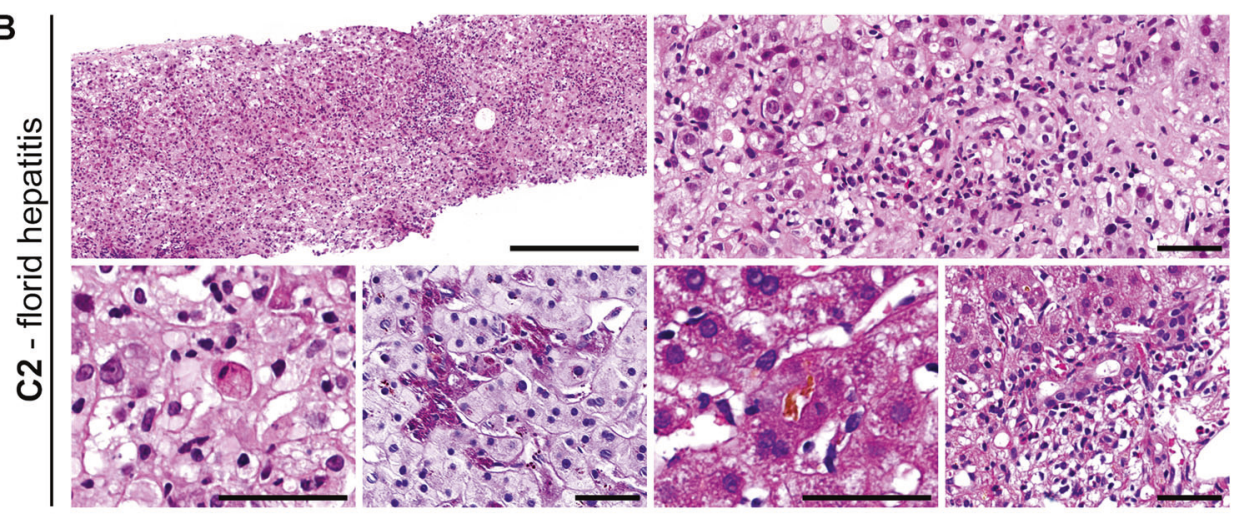

C

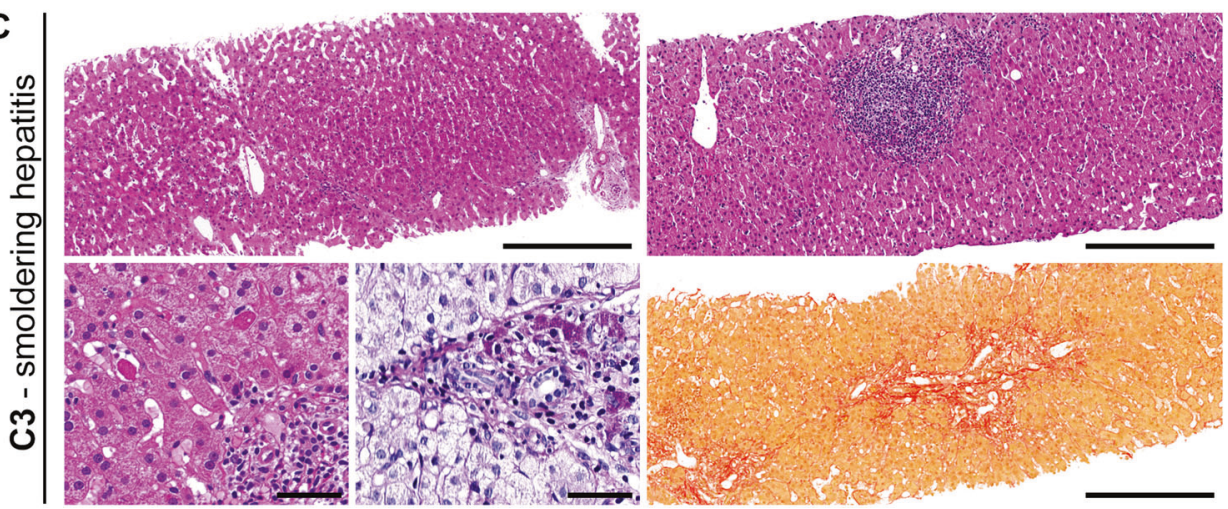

Samples in C1 showed mainly features of acute-on-chronic LD, such as cirrhosis, necrosis, steatosis, steatohepatitis, hepatocyte damage, Mallory-Denk hyaline and cholestasis (Fig. 3a). Samples in C2 displayed mainly features of florid hepatitis, such as lobular inflammation, parenchymal disarray, foci of hepatocyte necrosis and many scattered apoptotic hepatocytes as well as signs of beginning removal with lobular ceroid-laden histiocytes, cholestasis and bile duct damage (Fig. 3b). Samples in C3 were mainly characterized by features of smoldering hepatitis, ranging from minimal histologic changes over mild to more pronounced portal based inflammation, mostly with mild interface and lobular activity with only rare apoptotic hepatocytes, as well as signs of previous damage such as ceroid-laden histiocytes in portal tracts and in some cases with fibrosis (Fig. 3c).

Thus, similar as observed for the five histologic patterns, also the three clusters displayed a broad distribution among the cases of our cohort (Table 2 and Supplementary Fig. 4a), and were unequally associated with specific patient characteristics, some of them with statistical significance. For example, in C1 (acute-on-chronic LD) the group of patients with pre-existing LD was statistically significantly over-represented, whereas in $\mathrm{C} 2$ (florid hepatitis) the group of immunocompetent patients without pre-existing LD was statistically significantly over-represented. In contrast, in C3 

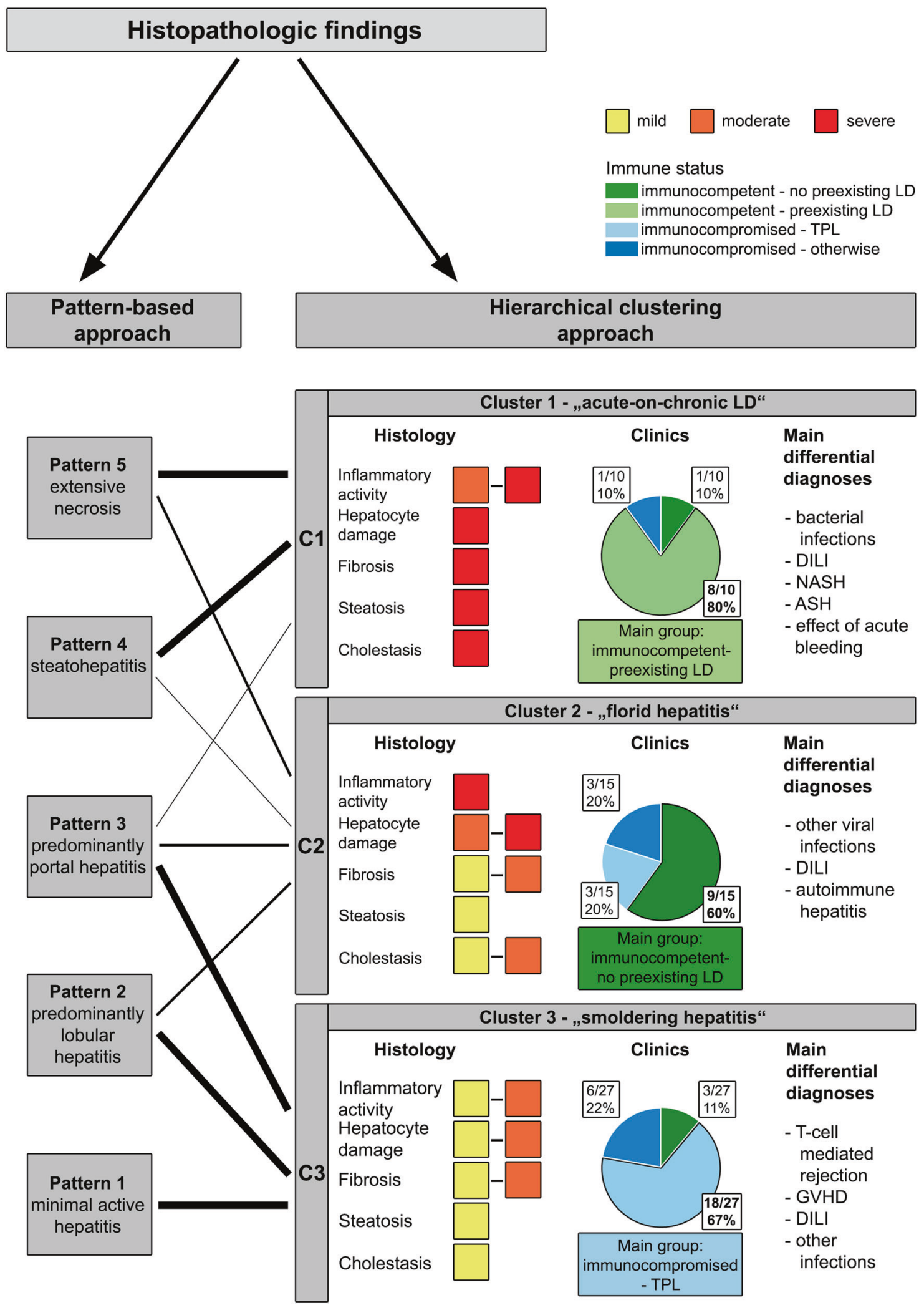

Fig. 4 Association of the three clusters with the clinical setting and the five histologic patterns. Clinico-pathologic settings of the three clusters including their most relevant differential diagnoses. The thickness of the lines between the patterns and the clusters represent the relative association of both. ASH alcoholic steatohepatitis, C cluster, DILI drug-induced liver injury, GVHD graft-versus-host disease, LD liver disease, NASH nonalcoholic steatohepatitis, TPL transplantation. 
(smoldering hepatitis), the group of immunocompetent patients without pre-existing LD was statistically significantly under-represented (Supplementary Fig. 4b).

Collectively, hierarchical clustering with respect to the 33 histopathologic features identified three histopathologic clusters, which showed distinct histopathologies, some of which were preferentially associated with specific patient characteristics.

\section{Three histopathologic clusters reflect different clinical scenarios}

Next, we sought to investigate the association between the three clusters $\mathrm{C} 1-\mathrm{C} 3$ identified by statistical cluster analysis methods, and the patients' immune status and preexisting liver condition, considered to be relevant for the management of patients with hepatitis E [4]. Overall, it turned out that each of the three clusters $\mathrm{C} 1-\mathrm{C} 3$ was dominated by one main patient group (Fig. 4): C1 representing acute-on-chronic liver disease was dominated by patients with pre-existing LD $(8 / 10 ; 80 \%)$; C2 representing florid hepatitis was dominated by immunocompetent patients without known pre-existing LD $(9 / 15 ; 60 \%)$; C3 representing smoldering hepatitis was dominated by immunocompromised transplanted patients $(18 / 27 ; 67 \%)$. Samples from patients immunocompromised for other reasons were found in all three clusters without a clear predominance. Of note, the five histologic patterns were represented differently in the three clusters (Fig. 4 and Supplementary Fig. 5a), and the three clusters were unequally associated with the five patterns, some of them with statistical significance. For example, the patterns "extensive necrosis" and "steatohepatitis" were statistically significantly over-represented in C1 (Supplementary Fig. 5b).

Moreover, we sought to investigate the association between the three clusters $\mathrm{C} 1-\mathrm{C} 3$ and the outcome with respect to the HEV infection. After 3 months as well as after 6 months, a strong positive association was found for $\mathrm{C} 1$ and "liver failure, death/liver failure, transplantation", and a strong negative association was found for $\mathrm{C} 3$ "liver failure, death/liver failure, transplantation." Furthermore, a strong positive association was found for $\mathrm{C} 2$ and "resolved infection", but only after 3 months (Table 2 and Supplementary Fig. 6).

Collectively, these findings not only show that the three histopathologic clusters are differentially associated with the five histologic patterns, but also suggest that they reflect different clinical scenarios, which seem to be defined by the patients' immune status and pre-existing liver condition, and also to affect the outcome of the HEV infection.

\section{Discussion}

The findings of our study provide a rational explanation for the well-known broad and at first sight puzzling spectrum of histopathologic changes observed in hepatitis E. These results were made possible by (1) the availability of a significant number of hepatitis $E$ tissue samples from patients with very different clinical backgrounds and (2) by pursuing a novel data-driven statistical approach. This allowed us to also conduct comparative studies on the different clinical settings of hepatitis $\mathrm{E}$, which goes beyond the previous reports on narrowly defined cohorts such as hepatitis $\mathrm{E}$ in epidemic outbreaks or in patients with immunosuppression.

With both methods, the traditional pattern-based approach as well as the cluster analysis, we were able to structure the broad histopathologic spectrum of hepatitis E represented in this cohort. Of note, we ultimately found overlapping results between the two different approaches. Nevertheless, a strength of the data-driven approach is its independence of any predetermined category. We therefore consider the resulting clustering to be a solid basis for the classification of hepatitis E histopathology.

Of particular interest is the first cluster $(\mathrm{C} 1$, acute-onchronic liver disease), which includes mainly patients with pre-existing LD. From a histopathologic perspective, it is noteworthy that the observed changes were determined primarily by the respective pre-existing liver disease, i.e., cirrhosis on the background of ASH or NASH. Of note, beside cholestatic changes, considered to reflect hepatic decompensation, HEV infection in these cases showed no additional characteristic or even specific histologic features. As the samples in $\mathrm{C} 1$ mostly showed advanced fibrosis or cirrhosis as well as signs of steatohepatitis, the most important histopathologic differential diagnosis in this group is acute (non)alcoholic hepatitis. However, from a clinical perspective the differential diagnosis is much broader, including other reasons for acute decompensation such as bacterial infections, drug-induced liver injury (DILI), complications of acute bleeding, etc. Five of seven patients in $\mathrm{C} 1$ had cirrhosis and eventually died as a result of hepatitis E, in line with the previously reported poor prognosis in this patient group [6,33]. A detailed clinical description of the patients with pre-existing LD who died in the context of HEV infection shall be reported elsewhere. Interestingly, the two patients in $\mathrm{C} 1$ who did not die (pat. 19 and 32), presented with only incomplete cirrhosis, presumably reflecting a higher reserve in coping with HEV infection. In conclusion for $\mathrm{C} 1$, knowledge and awareness of the specifics of (acute) hepatitis $\mathrm{E}$ in pre-existing liver disease is essential for both pathologists and clinicians. In daily practice, pronounced cholestatic changes and/or significantly elevated ALT values should prompt them to 
systematically look for reasons for liver decompensation, including hepatitis E [34].

The second cluster ( $\mathrm{C} 2$, florid hepatitis) revealed an overrepresentation of immunocompetent patients without any known pre-existing LD, but included also five patients under immunosuppression. The fact that those five patients presented with high inflammatory activity may point to a relatively mild immunosuppression at the time of HEV infection. Histology was dominated by highly active hepatitis. The three top differential diagnoses from a pathological as well as from a clinical point of view are other viral infections, DILI, and autoimmune hepatitis $[17,35]$.

The third cluster ( $\mathrm{C} 3$, smoldering hepatitis) included mainly patients under (probably more intense) immunosuppression. In line with an immune-mediated pathogenesis of hepatitis E, only mild to moderate inflammatory activity was observed in these patients. However, 13/22 patients in this group experienced a prolonged infection (3 months or longer, i.e., chronic hepatitis E), some of them also with developing fibrosis, which is in line with the current literature $[7,16]$. In two of the immunocompromised patients in C3, histology revealed also cirrhotic changes. Patient 22 with chronic lymphocytic leukemia under rituximab/lenalidomid treatment had chronic hepatitis E persisting for 4 years. The etiology of liver cirrhosis in this patient was possibly a combination of hepatitis E and NASH, for which hints were found in histology. Patient 23 with a history of rheumatoid arthritis may have developed cirrhosis due to methotrexate treatment. It is possible that the three patients classified as immunocompetent in $\mathrm{C} 3$ (pat. 10, 16, and 17) had a hitherto unrecognized immunodeficiency, and as a consequence developed less active inflammation. Alternatively, since the biopsies showed clear signs of subacute inflammation, they may also have been taken at a later stage of HEV infection. The most important differential diagnoses from a pathologic as well as from a clinical point of view are other (viral) infections and DILI, as well as (acute) T-cell mediated rejection in patients with solid organ TPL and graft-versus-host disease in patients with HSC TPL [17, 35, 36].

Interestingly, in one and the same patient, the histologic changes caused by hepatitis E can vary considerably depending on the time the biopsy was taken, which most likely reflects the variable intensity of the patients' immunosuppression. For example, three biopsies from a liver transplant patient (pat. 2) clustered in C3 early during infection, while two biopsies taken at a later point in time were found in $\mathrm{C} 2$.

In routine diagnostics, both the pathologist and the clinician are not confronted with particular entities but with their manifestations, from which they must deduce the distinct etiologies behind them. The findings of our study suggest that for histopathologic-as well as clinicaldiagnosis of hepatitis $\mathrm{E}$, it is advisable to consider the patients' immune status and pre-existing liver condition. This approach allows to sharpen and narrow down the differential diagnostic spectrum. For example, when being confronted with a little active pattern, hepatitis E should be among the top differential diagnoses in an immunocompromised patient, but it may be considered less likely in an immunocompetent individual.

Considering that the histopathologic changes in a tissue are a direct reflection of an organism's exposure to a harmful agent, such as an infectious particle, it is obvious that the histopathologic changes observed in relation to a particular disease are not only of interest to pathology but also provide insight into the biology of the disease, in particular with respect to immunologic aspects [37, 38]. We postulate that the associations of histologic patterns with the immune status and pre-existing liver condition of patients shown here reflect not only a random statistical association but have a causal link.

The cohort underlying this study is, to the best of our knowledge, the world's largest hepatitis E biopsy cohort comprising $>50$ specimens taken from patients with different immune and liver status, enriched with gt 3 infections [19]. With a predominance of HEV gt 3 and some travel associated gt 1 infections, it can be considered as representative not only for Europe, but also for North America where awareness of hepatitis E as a relevant health problem has increased recently [4, 39, 40]. Nevertheless, there are some potential limitations. First, despite the above mentioned high number of hepatitis $\mathrm{E}$ specimens, the sample size is still relatively low with respect to the applicability of confirmatory statistical analysis methods. Moreover, our results may not be generalizable to countries in which other gt predominate, such as Asia with gt 1 and gt 4 infections. However, it is conceivable that the histopathologic findings of cases of acuteon-chronic liver failure, as characterized here in $\mathrm{C} 1$, could be caused by gt 1 , gt 2 or gt 4 infection as well [41]. Other potential limitations are the disproportionately high number of hepatitis E cases with a prolonged/chronic course in immunocompromised patients, since these are biopsied in a higher proportion, and the retrospective setting without standardized conditions, especially for the time point of the biopsy procurement during the infection.

In summary, the results of our study provide a plausible explanation for the broad, only at first glance unstructured histopathologic spectrum observed in hepatitis E, and provide insight into the underlying determinants. From these findings we expect not only a better understanding of the biology of HEV infection, but also guidance to the pathologic and clinical diagnosis, which should improve the care of patients with hepatitis E. 
Acknowledgements We would like to thank Christine Mittmann, André Fitsche as well as Martina Storz and her team for their excellent technical assistance.

Funding This study was supported by grants from the Uniscientia Stiftung, Zurich and the University Hospital Zurich ("USZ Innovations-Pool") to AW.

\section{Compliance with ethical standards}

Conflict of interest The authors declare that they have no conflict of interest.

Publisher's note Springer Nature remains neutral with regard to jurisdictional claims in published maps and institutional affiliations.

\section{References}

1. Debing Y, Moradpour D, Neyts J, Gouttenoire J. Update on hepatitis $\mathrm{E}$ virology: implications for clinical practice. J Hepatol. 2016;65:200-12.

2. Nimgaonkar I, Ding Q, Schwartz RE, Ploss A. Hepatitis E virus: advances and challenges. Nat Rev Gastroenterol Hepatol. 2018; 15:96-110.

3. Kamar N, Izopet J, Pavio N, Aggarwal R, Labrique A, Wedemeyer H, et al. Hepatitis E virus infection. Nat Rev Dis Prim. 2017;3:17086.

4. European Association for the Study of the Liver. EASL clinical practice guidelines on hepatitis $\mathrm{E}$ virus infection. J Hepatol. 2018;68:1256-71.

5. Dalton HR, Izopet J. Transmission and epidemiology of hepatitis E virus genotype 3 and 4 infections. Cold Spring Harb Perspect Med. 2018;8:a032144.

6. Dalton HR. Hepatitis: hepatitis E and decompensated chronic liver disease. Nat Rev Gastroenterol Hepatol. 2012;9:430-2.

7. Kamar N, Selves J, Mansuy JM, Ouezzani L, Péron JM, Guitard J, et al. Hepatitis $\mathrm{E}$ virus and chronic hepatitis in organ-transplant recipients. N Engl J Med. 2008;358:811-7.

8. Viswanathan R. Epidemiology. Indian J Med Res. 1957;45:1-29.

9. Gupta DN, Smetana HF. The histopathology of viral hepatitis as seen in the Delhi epidemic (1955-56). Indian J Med Res. 1957; 45:101-13

10. Peron JM, Danjoux M, Kamar N, Missoury R, Poirson H, Vinel JP, et al. Liver histology in patients with sporadic acute hepatitis E: a study of 11 patients from South-West France. Virchows Arch. 2007;450:405-10.

11. Malcolm P, Dalton H, Hussaini HS, Mathew J. The histology of acute autochthonous hepatitis E virus infection. Histopathology. 2007;51:190-4.

12. Drebber U, Odenthal M, Aberle SW, Winkel N, Wedemeyer I, Hemberger J, et al. Hepatitis E in liver biopsies from patients with acute hepatitis of clinically unexplained origin. Front Physiol. 2013;4:351.

13. Prost S, Crossan CL, Dalton HR, De Man RA, Kamar N, Selves J, et al. Detection of viral hepatitis $\mathrm{E}$ in clinical liver biopsies. Histopathology. 2017;71:580-90.

14. Protzer U, Böhm F, Longerich T, Seebach J, Heidary Navid M, Friemel J, et al. Molecular detection of hepatitis E virus (HEV) in liver biopsies after liver transplantation. Mod Pathol. 2015;28: 523-32.

15. Beer A, Holzmann H, Pischke S, Behrendt P, Wrba F, Schlue J, et al. Chronic Hepatitis $\mathrm{E}$ is associated with cholangitis. Liver Int. 2019;39:1876-83.
16. Kamar N, Garrouste C, Haagsma EB, Garrique V, Pischke S, Chauvet $\mathrm{C}$, et al. Factors associated with chronic hepatitis in patients with hepatitis $\mathrm{E}$ virus infection who have received solid organ transplants. Gastroenterology. 2011;140:1481-9.

17. Davern TJ, Chalasani N, Fontana RJ, Hayashi PH, Protiva P, Kleiner DE, et al. Acute hepatitis E infection accounts for some cases of suspected drug-induced liver injury. Gastroenterology. 2011;141:1665-72. e1-9

18. Chijioke O, Bawohl M, Springer E, Weber A. Hepatitis e virus detection in liver tissue from patients with suspected drug-induced liver injury. Front Med (Lausanne). 2015;2:20.

19. Cullen JM, Lemon SM. Comparative pathology of hepatitis A virus and hepatitis E virus infection. Cold Spring Harb Perspect Med. 2019;9:a033456.

20. Brunt EM, Janney CG, Di Bisceglie AM, Neuschwander-Tetri BA, Bacon BR. Nonalcoholic steatohepatitis: a proposal for grading and staging the histological lesions. Am J Gastroenterol. 1999;94:2467-74.

21. Bedossa P, Poynard T. An algorithm for the grading of activity in chronic hepatitis C. The METAVIR Cooperative Study Group. Hepatology. 1996;24:289-93.

22. Batts KP, Ludwig J. Chronic hepatitis. An update on terminology and reporting. Am J Surg Pathol. 1995;19:1409-17.

23. Ishak K, Baptista A, Bianchi L, Callea F, De Groote J, Gudat F, et al. Histological grading and staging of chronic hepatitis. J Hepatol. 1995;22:696-9.

24. Kirkwood B, Sterne J. Essential medical statistics. Oxford: WileyBlackwell; 2003. chapter 36.3, p. 433-7.

25. Kaufman L, Rousseeuw R. Finding groups in data: an Introduction to Cluster Analysis. New York: Wiley; 1990. chapter 2.6, p. 32-7; chapter 6, p. 253-9.

26. R_Core_Team. A Language and Environment for Statistical Computing. R Foundation for Statistical Computing. https://www. R-project.org/. 2020.

27. Falissard B psy: various procedures used in psychometry. R package version 1.1. https://CRAN.R-project.org/package=psy. 2012.

28. Maechler M, Rousseeuw P, Struyf A, Hubert M, Hornik K. Cluster: cluster analysis basics and extensions. R package version 2.1.0. 2019.

29. Warnes G, Bolker B, Bonebakker L, Gentleman R, Liaw A, Lumley $\mathrm{T}$, et al. gplots: Various $\mathrm{R}$ Programming Tools for Plotting Data. R package version 3.0.3. https://CRAN.R-project. org/package $=$ gplots. 2020.

30. Zeileis A, Meyer D, Hornik K. Residual-based shadings for visualizing (conditional) independence. J Comput Graph Stat. 2007;16:507-25.

31. Lenggenhager D, Gouttenoire J, Malehmir M, Bawohl M, Honcharova-Biletska $\mathrm{H}$, Kreutzer $\mathrm{S}$, et al. Visualization of hepatitis E virus RNA and proteins in the human liver. J Hepatol. 2017;67:471-9.

32. Romil S. Practical hepatic pathology: a diagnostic approach. 2nd ed. Philadelphia: Elsevier; 2018. Preface, p. XXi-XXiii.

33. Kumar Acharya S, Kumar Sharma P, Singh R, Kumar Mohanty S, Madan K, Kumar Jha J, et al. Hepatitis E virus (HEV) infection in patients with cirrhosis is associated with rapid decompensation and death. J Hepatol. 2007;46:387-94.

34. Altamirano J, Miquel R, Katoonizadeh A, Abraldes JG, DuarteRojo A, Louvet A, et al. A histologic scoring system for prognosis of patients with alcoholic hepatitis. Gastroenterology. 2014;146:1231-9. e1-6

35. Lenggenhager D, Weber A. An update on the clinicopathologic features and pathologic diagnosis of hepatitis $\mathrm{E}$ in liver specimens. Adv Anat Pathol. 2018;25:273-81.

36. Demetris AJ, Bellamy C, Hübscher SG, O'Leary J, Randhawa PS, Feng S, et al. 2016 comprehensive update of the banff working 
group on liver allograft pathology: introduction of antibodymediated rejection. Am J Transpl. 2016;16:2816-35.

37. Walker CM. Adaptive immune responses in hepatitis A virus and hepatitis E virus infections. Cold Spring Harb Perspect Med. 2019;9:a033472.

38. Feng Z, Lemon SM. Innate immunity to enteric hepatitis viruses. Cold Spring Harb Perspect Med. 2019;9:a033464.

39. Horvatits T, Ozga AK, Westhölter D, Hartl J, Manthey CF, Lütgehetmann $\mathrm{M}$, et al. Hepatitis $\mathrm{E}$ seroprevalence in the Americas: a systematic review and meta-analysis. Liver Int. 2018;38:1951-64.

40. Whitsett M, Feldman DM, Jacobson I. Hepatitis E Virus infection in the United States: current understanding of the prevalence and significance in the liver transplant patient population and proposed diagnostic and treatment strategies. Liver Transpl. 2020;26:709-17.

41. Wang Y, Liu H, Liu S, Yang C, Jiang Y, Wang S, et al. Incidence, predictors and prognosis of genotype 4 hepatitis $\mathrm{E}$ related liver failure: a tertiary nested case-control study. Liver Int. 2019;39:2291-300.

42. Friedman LS, Lee SR, Nelson SB, Masia R. Case 36-2016. A 50Year-Old Man With Acute Liver Injury. N Engl J Med. 2016; 375:2082-92.

43. Lenggenhager D, Weber A. Hepatitis E Virus and the Liver: Clinical Settings and Liver Pathology. Gastroenterol Clin North Am. 2017;46:393-407. 